\title{
Experiment into Nondestructive Testing of Rail Foot Cracks Using Infrared Thermal Waves
}

\author{
JIA Wenjing \\ (School of Automation and Electrical Engineering, Huainan Normal University, Huainan 232038, China)
}

\begin{abstract}
At present, ultrasonic testing is widely used in rail crack detection. However, in the process of rail crack detection, there is a certain blind spot in this method, and cracks on both sides of the rail foot are not detected. Aiming at these shortcomings, an infrared thermal wave nondestructive testing platform for detecting cracks on the rail foot is proposed in this paper. Infrared thermal wave nondestructive testing for rail cracks is a new research area in the field of infrared nondestructive testing, and the establishment of the experimental platform mainly includes three parts: the selection of the thermal excitation source, the construction of the experimental platform, and the extraction of the infrared images. The technology is used to heat the surface of the rail foot through a thermal excitation source. According to the wave theory of the heat transfer from the surface to the inside of the rail foot, the transfer process can be affected by the rail's physical characteristics and internal structure. If there are defects inside the rail, the distribution of the temperature on the rail surface will be affected according to the type of defect. The location of the internal defects of the rail can be determined according to the distribution of the surface temperature information of the rail, thereby achieving the goal of crack detection in the rail. Experimental results show that the surface temperature of the rail foot corresponding to the crack is higher than the corresponding rail foot surface temperature without cracks, proving that infrared thermal wave NDT(non-destructive testing) is feasible for rail crack detection.
\end{abstract}

Key words: rail foot crack in rail, infrared thermal wave nondestructive testing, thermal imager, infrared image

\section{钢轨轨脚裂纹红外热波无损检测实验}

\author{
贾文晶 \\ (淮南师范学院 机械与电气工程学院, 安徽 淮南 232038)
}

\begin{abstract}
摘要: 目前, 超声检测在钢轨裂纹检测中得到了广泛的应用, 但在钢轨裂纹检测过程中, 超声波检 测方法存在一定的盲区，未能检测到轨脚两侧的裂纹。针对超声波检测方法的不足，搭建了一种红 外热波钢轨踏面裂纹无损检测平台。钢轨裂纹红外热波无损检测平台是红外无损检测领域的一个新 的研究方向, 实验平台的建立主要包括 3 部分: 热激励源的选择、实验平台的搭建、红外图像的提 取。该技术用于通过热激励源加热轨底表面。根据热波理论, 钢轨表面到轨底内侧的传热过程受钢 轨的物理特性和内部结构的影响, 如果钢轨内部存在缺陷, 则缺陷类型会影响钢轨表面的温度分布。 根据钢轨表面温度信息的分布, 确定钢轨内部缺陷的位置, 达到检测钢轨裂纹的目的。实验结果表 明, 裂纹对应的轨底表面温度高于无裂纹对应的轨底表面温度, 证明红外热波无损检测对钢轨裂纹 检测是可行的。
\end{abstract}

关键词: 轨脚裂纹; 红外热波无损检测; 热像仪; 红外图像

中图分类号：TP231 文献标识码：A 文章编号：1001-8891(2020)02-0163-05

\section{Introduction}

Railway transportation is a national economic artery and an indispensable part of social and economic development. Due to its characteristics of safety, economy, high speed, and large capacity, railway

Receiving date: 2018-11-08; Revision date: 2019-12-10.

Brief introduction of the author: Jia Wenjing (1991-), Female, teaching assistant, mainly engaged in image processing and fault diagnosis. E-mail: jiawen 0621@163.com。

Fund projects: General scientific research projects of Huainan Normal University(2018xj33). 
transportation is the backbone of China's comprehensive transportation system. In addition, it plays an important role in the development of the social economy and the improvement of people's lives. Rails are an essential part of the railway operation, and monitoring them for damage is vital to ensure safe operation of the trains ${ }^{[1]}$.

Since 1997, China's railway operation speed has been upgraded six times. At present, train operation speeds on the main trunk lines of China's railway can reach 200 $\mathrm{km} / \mathrm{h}$, and on some routes this can reach $250 \mathrm{~km} / \mathrm{h}$. As a result, China's railway transportation system has stepped into the era of high-speed operation. With the continuous improvement of train speed, ensuring train safety has become the top priority for railway development. Although railway departments actively promote the laying of CWR(continuous welded rail), rail joints are still used near buffer sections and turnout areas of many trunk lines, which are not smooth. When a high-speed train passes through an area of track irregularity, the huge impact of the wheels on the track causes many new forms of rail cracking, which is a serious threat to the safety of railway operation. At present, ultrasonic testing is the most widely used rail crack detection method in China's railways. In the ultrasonic testing method, whether a large rail inspection vehicle or a small manual reinspection vehicle is used, there is a certain blind spot in the detection of rail bottom cracks, and cracks on both sides of the rail foot cannot be detected. During rail crack detection, the ultrasonic probe on the rail detection vehicle sends out ultrasonic signals from the rail surface inlet. Although the ultrasonic detection speed and precision is high, the ultrasonic detection range is \pm 10 $\mathrm{mm}$ in the center axis of the track, and defects inside the two sides of the rail cannot be detected. To meet people's needs for safe railway operation and to ensure that the train will not break or derail while running, the detection of rail bottom cracks has received increasing attention from railway departments. Hence, it is of great theoretical significance and practical value to carry out research into infrared thermal wave NDT(non-destructive testing) of rail bottom cracks ${ }^{[2]}$.

\section{Infrared thermal imager}

An infrared thermal imager is a device for detecting infrared thermal radiation and the surface temperature distribution of objects. According to blackbody theory, except for an object at absolute zero $\left(-273^{\circ} \mathrm{C}\right)$, any object will emit infrared radiation. The infrared thermal imager detects the radiation emitted by the object through an infrared sensor and generates an infrared thermal image. An infrared thermal imager can determine the internal structure of the object by analyzing the temperature information on the surface of the object, which can then be used to establish whether the interior of the object is defective. At present, the detection accuracy of infrared thermography is up to $0.02^{\circ} \mathrm{C}$. In theory, provided the temperature distribution of the rail surface is more than $0.02^{\circ} \mathrm{C}$, the infrared thermal imager can collect the temperature distribution information from the surface of the rail foot, thus realizing the objective of detecting any internal cracks ${ }^{[3]}$.

An infrared thermal imager mainly consists of five parts: the lens, infrared detector, electronic components, software system, and the display element. The infrared thermal imager takes pictures of the object through the lens, and the infrared detector can detect infrared thermal radiation emitted by the object. Electronic components then convert the detected infrared thermal radiation into electrical signals. The software system can transform the electrical signal into an infrared thermal image with temperature information, and finally display the infrared image through the display element.

An infrared thermograph has better controllability compared with other imaging equipment. It can be connected to a computer by IEEE-1394 to realize the output and storage of the infrared thermal image. Moreover, it has powerful data processing capability and can process 16-bit digital image information in real-time. Compared with image processing equipment, an infrared thermal imager has better measurement accuracy. The measurement range is between 40 and $150^{\circ} \mathrm{C}$, and the accuracy of temperature measurement is better than $0.02^{\circ} \mathrm{C}$. The infrared thermal images collected are extremely high resolution, and each infrared thermal image can reach 110592 pixels. An infrared thermal imager also has two additional capabilities after completing infrared thermal image acquisition. At present, an advanced infrared thermal image analysis system does not need to connect to a computer and can complete the analysis of the infrared thermal image by itself $^{[4]}$.

The infrared thermal imager used in this paper is the fotric 220 series thermal imager provided by Shanghai Juge company. The technical parameters of the infrared thermal imager are shown in Table 1.

\section{Heat sources}

It is essential to carefully select the thermal excitation source used in the infrared thermal wave NDT of active rail bottom cracks. Different thermal excitation sources 
are selected to heat the rail bottom surface, and an infrared thermal imager is used to collect the thermal wave signal from it. According to the temperature distribution of the rail bottom surface, an appropriate thermal excitation source is selected. Traditional thermal excitation sources include high-energy flash lamps, U-type xenon flash lamps, lasers, halogen lamps, and hot air. A halogen lamp is shown in Figure 1, and a U-type xenon flash lamp is shown in Figure 2.

Table 1 Technical parameters of FOTRIC 220 series infrared thermography

\begin{tabular}{lc}
\hline \multicolumn{1}{c}{ Technical indicators } & Specific parameters \\
\hline Minimum focal length (FOV) & $28 \mathrm{~mm} \times 21 \mathrm{~mm}$ \\
Spatial resolution(IFOV) & $1.27 \mathrm{mrad}$ (standard lens) \\
Thermal sensitivity/NETD & $<0.05^{\circ} \mathrm{C}$ \\
Focus mode & Manual focusing \\
Wavelength range & $8-14 \mu \mathrm{m}$ \\
Frame rate & $50 / 60 \mathrm{~Hz}$ \\
Temperature range & $0-150^{\circ} \mathrm{C}$ \\
\hline
\end{tabular}

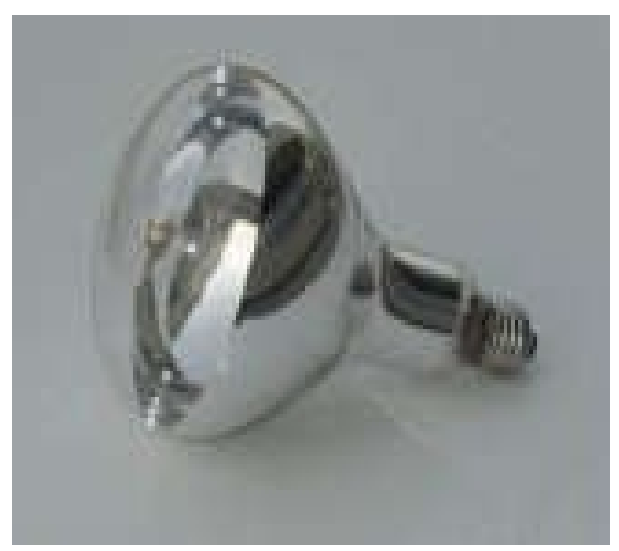

Fig. 1 Halogen lamp

The infrared image obtained by heating with the halogen lamp is shown in Figure 3, and that obtained by heating with the U-type xenon flash is shown in Figure 4. The crack center temperature can reach $25.9{ }^{\circ} \mathrm{C}$ using a U-type xenon flash lamp as the thermal excitation source, and $38.2{ }^{\circ} \mathrm{C}$ using a halogen lamp.

By comparing and analyzing the heating effect of the two kinds of thermal excitation source, such as the heating time required for the rail foot surface to reach the same temperature and the temperature achieved at the rail foot surface with the same time, it can be shown that the requirements for infrared thermal wave nondestructive testing of active rail bottom cracks can be met using the halogen lamp as the thermal excitation source. Compared with the U-type xenon flash lamp, it can operate at a higher temperature, and the efficiency in heating the rail foot surface as a thermal excitation source is higher. A halogen lamp only transforms $6 \%$ to $10 \%$ of electrical energy into light energy, and the remaining part is converted into heat energy. Moreover, a halogen lamp has the advantages of low cost, long service life, good seismic resistance, and easy heat control, which is more suitable for the infrared thermal wave detection system of rail bottom cracks. The halogen lamp can transfer different heat energies according to the length of the heating time. The maximum energy of the halogen lamp is $4500 \mathrm{~J}$, and the minimum energy is $700 \mathrm{~J}^{[5]}$.

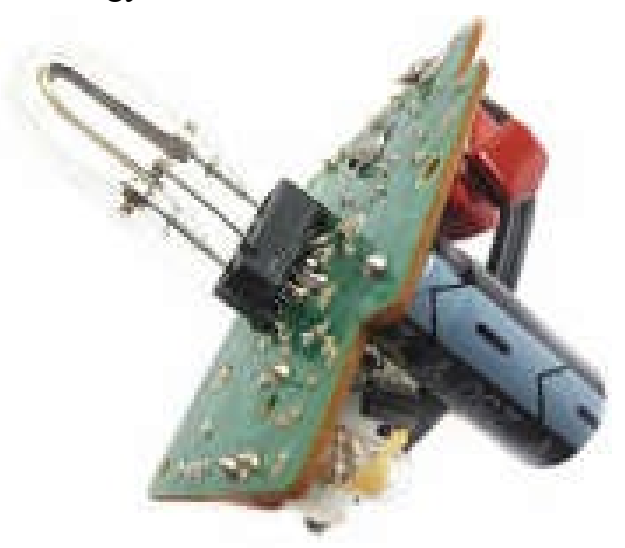

Fig. 2 U-type xenon flash lamp

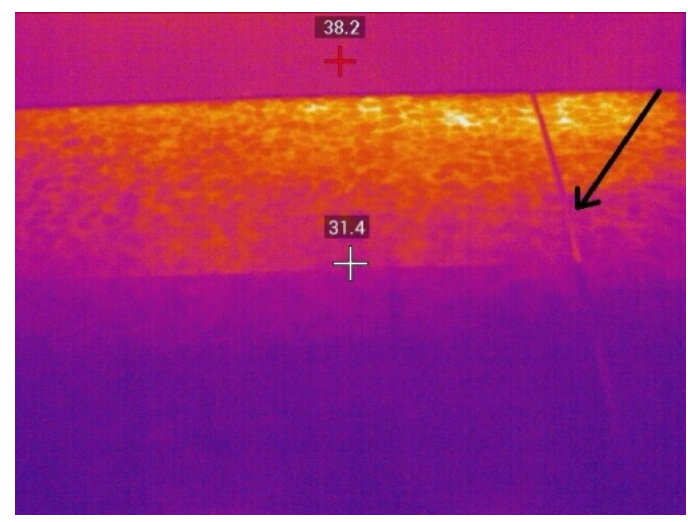

Fig. 3 Infrared image of halogen lamp heating

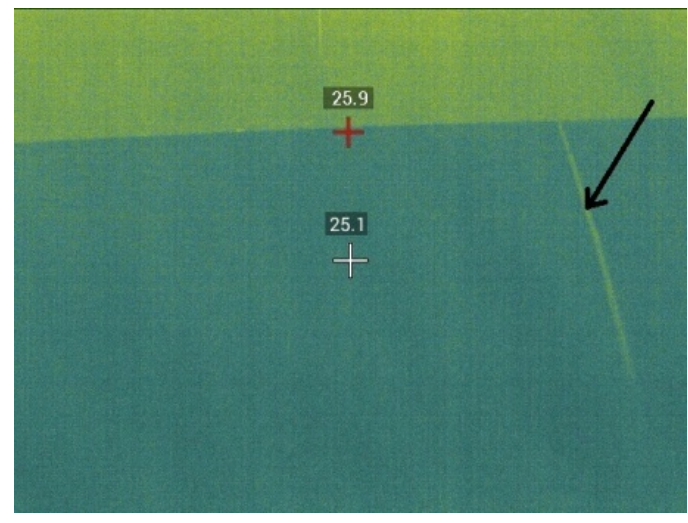

Fig.4 Infrared image heated by U-type xenon flash 


\section{Experimental platform construction}

The active infrared thermal wave nondestructive testing system is mainly used to complete the detection of rail bottom cracks. The whole system consists of three parts: the thermal excitation source (halogen lamp), the image processing system (computer analysis software), and the image acquisition system (infrared thermal imager) ${ }^{[5]}$.

The experimental block diagram is shown in Figure 5. The detection process of the system uses a $1000 \mathrm{~W}$ halogen lamp for actively heating the rail foot surface, and the heat flow transfers from the surface to the rail interior. After heating is completed, an infrared thermal image of the rail foot surface is collected by the infrared thermal imager. The infrared thermal image of the rail foot crack collected by the online infrared image analysis software in the computer is processed according to the temperature in the thermal image and is used to determine whether there are defects in the rail foot.

As shown in Figure 5, an experimental platform for non-destructive infrared thermal wave detection of rail foot cracks in active rails has been built, and the procedural steps for the experimental platform are as follows:

- First, the foot surface of the rail should be treated, and dust and rust on the rail foot surface should be ground clean.

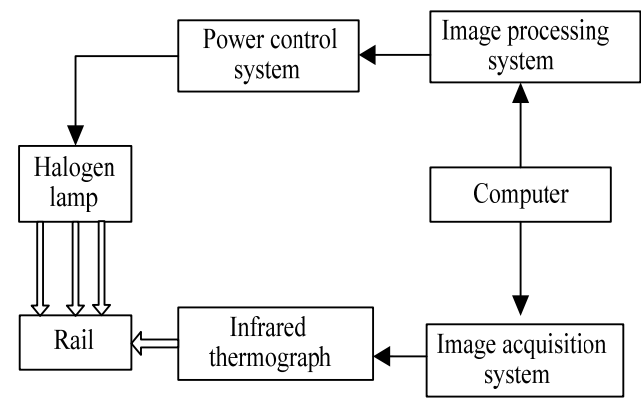

Fig.5 Block diagram of infrared thermal wave nondestructive testing of rail foot crack in active rail

- Installation of halogen lamps. Hang two $1000 \mathrm{~W}$ halogen lamps on the lamp cylinder pole, erect a tripod at each end of the lamp cylinder pole and fix the lamp cylinder pole across it. Adjust the height of the tripods and the angle of the halogen lamps to aim the halogen lamps at the surface of the rail foot, at the crack.

-Set-up of the infrared thermal imager. The cracks are detected by an ordinary lens and two macro lenses (50 and $100 \mathrm{~mm}$ ). The quality of the image acquisition is closely related to the location of the infrared thermal imager and the selection of the lens.

- Finally, the infrared thermal imager is connected to the computer, the on-line infrared thermal image analysis software is opened, and the collection of the infrared image is started. The infrared thermal imager requires heating by a thermal shock source for $5 \mathrm{~s}$ before collecting infrared images.

After the set-up of the infrared thermal wave nondestructive testing system for rail bottom cracks, the system needs to be debugged. First, turn on the thermal imager and start the on-line infrared thermal image analysis software on the computer. When the thermal imager is in a stable working mode, observe whether the thermal image displayed by the computer in real-time meets the requirements of the rail bottom crack detection experiment. According to the different detection methods used, the infrared thermal wave nondestructive testing experiment for rail bottom cracks can be split into single-sided and double-sided detection methods. The single-sided detection method has the thermal excitation source and the infrared thermal imager on the same side of the rail. The double-sided detection method has the thermal excitation source and the infrared thermal imager on opposite sides of the rail. However, due to the real-time requirements of rail bottom crack detection, the thermal imager and the thermal excitation source must be on the same side. In this paper, the single-sided detection method is used to collect the infrared thermal images of rail bottom cracks. The experimental platform for infrared thermal wave nondestructive testing of rail bottom cracks based on this active method is shown in Figure 6.

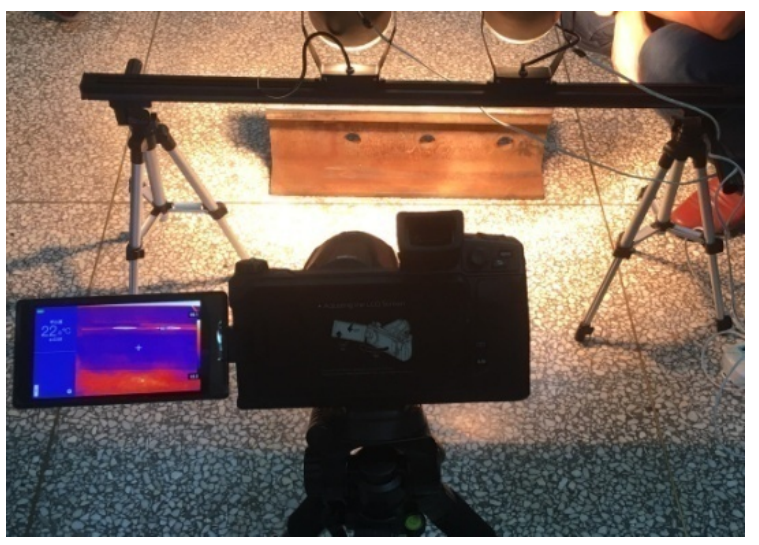

Fig.6 Experimental photo of infrared thermal wave nondestructive testing of rail foot cracks

\section{Infrared image acquisition and analysis}

\subsection{Infrared image acquisition}

The process of collecting the infrared thermal image of the rail bottom crack is started by turning on the power supply of the halogen lamp and letting the halogen lamp start heating. Then the infrared thermal image meter and 
computer are turned on after heating for $5 \mathrm{~s}$. Next, the infrared thermal image of the rail foot surface collected by the infrared thermal imager is transmitted to the computer. The time taken to collect the thermal image by the infrared thermal imager is $2 \mathrm{~s}$. There are two points to note in the infrared image acquisition process of the rail bottom cracks. First, it is necessary to locate the crack position with the tip of a pen and then fix the external thermal imager on the bracket according to the pen-tip position to get the best infrared thermal imager position. Second, due to corrosion of the rail foot surface caused by long-term use of the rail, the surface photothermal refractive index varies. Black ink is therefore applied to the rail foot surface to reduce its refractive index ${ }^{[6]}$. The process of rail heating and cooling was photographed during the experiment, and results showed that the effect of rail heating is better using this method. The experimental results show that the best quality infrared image is obtained when the lens is $9 \mathrm{~cm}$ away from the rail foot surface.

\subsection{Infrared image analysis}

The infrared image of the rail crack was analyzed online. ThermoX infrared image online analysis software was opened on the computer, the infrared image of the rail foot crack was input, and the temperature distribution in the image was analyzed. The interface screenshot of the online rail crack infrared image analysis software is shown in Figure 7.

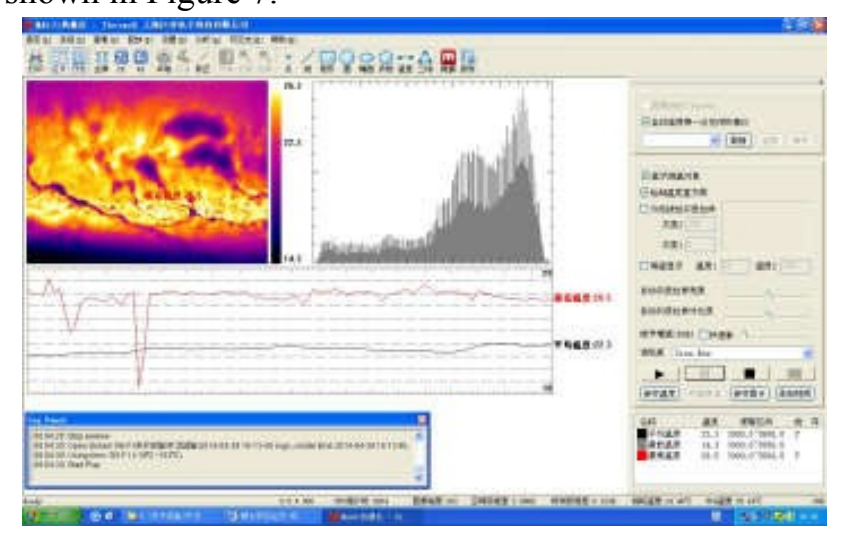

Fig.7 Interface screenshot of rail foot crack infrared image analysis software

The results of the on-line analysis of the rail foot crack infrared images are also shown in Figure 7. The upper left corner of the image shows the infrared image video and dynamic histogram. From this module, it can be seen that the highest temperature point is at the crack in the rail bottom. Over the whole image, the highest temperature point reached $26.7^{\circ} \mathrm{C}$, and the lowest temperature point was $14.1^{\circ} \mathrm{C}$. Looking at the temperature distribution information in the image, it is easy to determine the position and shape of the internal crack in the rail foot. The upper right corner of the figure is the set-up control area for the thermal imager, where it is possible to choose the interface mode between the thermal imager and the computer. The lower-left corner of the image is the real-time temperature curve of the rail bottom crack image. The highest temperature value of the real-time curve shown in red is $26.7^{\circ} \mathrm{C}$, and the average temperature value of the real-time curve shown in black is $22^{\circ} \mathrm{C}$, The lower right corner of the image is the image saving control area. This module can set the brightness and contrast of the image and set the temperature curve to be displayed.

\section{Summary}

A test platform for rail foot crack detection based on infrared thermal wave NDT was established, and a halogen lamp was used as the thermal excitation source after experimental comparison. The surface of the rail foot is heated by the halogen lamp, and infrared thermal images are captured by an infrared thermal imager. Infrared image analysis software is then used to analyze the rail crack images. Through this analysis, the temperature of the rail surface corresponding to the crack is higher than the surface temperature of the rail without a crack. The experimental results agree with simulation results, indicating that this infrared nondestructive testing technology for rail crack detection is feasible.

\section{References:}

[1] LI Lin. Research on the development and development trend of various modes of transportation[J]. Heilongjiang Traffic Technology, 2012(7): 152-153.

[2] WANG Shan, SUN Quanxin. Study on safety system of high speed railway[J]. Inner Mongolia Science and Technology and Economy, 2003(4): 69-71.

[3] Sophian A, Edwards R S, TIAN G Y, et al. Dual-probe methods using pulsed eddy currents and electromagnetic acoustic transducers for NDT inspection[J]. Or Insight, 2007, 47(6): 341-345.

[4] WU Zongfan. Principle and technology development of infrared thermal imager[J]. Modern Science Instruments, 1997(2): 28-30.

[5] WU Yongqiang. Design and technology of low voltage halogen lamps[J]. Vacuum Electronic Technology, 2002(6): 47-48.

[6] HUANG Tao. Quantitative detection of rail fatigue crack depth based on infrared thermal wave technology[D]. Lanzhou: Lanzhou JiaoTong University, 2015: 19-27. 